\title{
IMAGERIE DES VOIES SEMINALES
}

\author{
C. Roy \\ Service de Radiologie A, Pavillon Chirurgical A, Hôpital Universitaire de Strasbourg \\ 1, place de l'Hôpital - 67091 Strasbourg Cedex
}

\section{ULTRASONOGRAPHY OF THE MALE REPRODUCTIVE TRACT.} During the past decade, various imaging method have been developed which can be used to study the male reproductive tract. Ultrasonography, especially using high frequencies $(7.5$ or $10 \mathrm{MHz}$ ) and transrectal probes, provides very good visualization of the prostatic and testicular parenchyma. This has allowed the diagnosis of two types of tissue : cysts, in the case of congenital disease or post-infection dilation : and calcification, as a sequela of post-infection disease. Although this approach permits an accurate assessment when there is a small mass, it is less precise for large masses, which require a conventional radiological approach. Cystic puncture provides fluid samples for analysis. The diagnosis of varicocoeles is greatly facilitated, but for localization of an ectopic testis ultrasonography is only accurate in the superficial inguinal region. Vasography remains the best method for evaluation of the vas deferens, but it does require a surgical approach with the attendant risk of vasal occlusion. It is essential that a complex congenital pathology be evaluated fully before contemplating surgery. Computer-assisted tomography (CAT scan) and magnetic resonance imaging (MRI) are only useful for ectopic testes, although MRI is the preferred method for investigating a complex congenital pathology dues to its multiplanar sections and good contrast between different tissues. Key words : Ultrasonography, transrectal ultrasonography, magnetic resonance imaging, MRI, prostate, testis, seminal vesicle. Andrologie, 1992, 2 : 70-74.

La voie séminale peut être assimilée à un fin canal compris entre 2 glandes sexuelles.Il y a encore peu d'années, l'imagerie médicale se limitait à la radiologie conventionnelle et n'était pas concernée par l'étude des bourses. Le radiologue se contentait de les protéger par un écran plombé, chez les patients jeunes, quand elles se trouvaient dans le champ du rayonnement. Depuis un peu plus d'une décennie, les méthodes d'imagerie médicale pour l'exploration des organes génitaux se sont multipliées. L'examen échographique (EC), surtout grâce au développement des sondes de haute fréquence et endocavitaires, permet une approche morphologique très précise des parenchymes. Mais il n'explore pas les structures canalaires dont l'analyse est encore du domaine de la radiologie conventionnelle. Les méthodes d'imagerie dites "lourdes", tomodensitomètrie (TDM) et imagerie par résonance magnétique (IRM) ont des indications plus limitées.

Nous aborderons les différentes méthodes d'imagerie puis leur rôle respectif dans les circonstances pathologiques.

\section{TECHNIQUES D'IMAGERIE MEDICALE POUR L'EXPLORATION DES VOIES SEMINALES}

Examen échographique

Examen de première intention, il ne sera performant que si les impératifs techniques suivants sont respectés. L'étude des bourses nécessite l'utilisation d'une sonde de haute fréquence : 7,5 ou $10 \mathrm{MHz}$, sauf en cas de grosse bourse, où on préfèrera une sonde de $5 \mathrm{MHz}$, une étude comparative et la réalisation de manoeuvres dynamiques (Valsalva et orthostatisme) en cas de pathologie veineuse. De façon exhaustive, on distingue 3 voies d'abord différentes pour l'exploration de la prostate : la voie sus-pubienne, la voie périnéale, la voie endorectale. Les techniques les plus couramment utilisées sont la voie suspubienne et endorectale. Elles sont complémentaires et toutes deux indispensables à l'exploration complète du complexe prostatovésiculaire. Pour l'exploration de la prostate, tous les résultats doivent être confrontés au toucher rectal. A l'inverse pour l'exploration des vésicules séminales, l'imagerie est fondamentale car l'examen clinique est peu performant en dehors des cas de très grosses masses. La voie endorectale nécessite l'introduction dans l'ampoule rectale de la sonde de fréquence élevée $(7,5 \mathrm{MHz})$ ou $5 \mathrm{MHz}$ en cas de grosse prostate. Cette voie est certes plus invasive, mais un opérateur entrainé peut réaliser l'examen sans gros inconfort pour le patient. Les contre-indications relatives sont les lésions ano-rectales ou une prostatite aigue. Cette voie permet l'analyse la plus précise de la structure de la prostate et des voies séminales adjacentes. Bien que la proximité de la prostate soit en théorie intéressante, la voie périnéale réalisée avec la même sonde que la voie suspubienne est très décevante.

\section{Résultats normaux (4):}

Le testicule apparaît comme une formation oblongue d'échogénicité moyenne et homogène. Il est entouré par une mince bande hyperéchogène pouvant représenter le feuillet viscéral de la vaginale ou l'albuginée. Le carrefour vasculotubulaire (corps d'Highmore) est facilement identifié dans 3/4 des cas sous forme d'une mince structure hyperéchogène linéaire ou plus souvent triangulaire à base externe postéro-supérieure et au sommet interne descendant vers le centre de la glande. La tête de l'épididyme, toujours visible, est une structure ronde ou triangulaire inférieure ou égale à $1 \mathrm{~cm}$, échogène à hyperéchogène, plus souvent homogène que légèrement hétérogène. Elle est au contact du pôle supérieur du testicule dont elle est séparée par un sillon anéchogène. (Fig.1). Les plexus veineux spermatiques postérieurs et surtout antérieurs situés en arrière de l'épididyme sont le plus souvent visibles à gauche. Il s'agit de formations anéchogènes tubulaires et serpigineuses. L'échographie Döppler couleur permet d'étudier les systèmes artériels et veineux du cordon et leur distribution dans le testicule, l'épididyme et les enveloppes testiculaires.

L'examen échographique de la prostate fournit 3 types de renseignements :

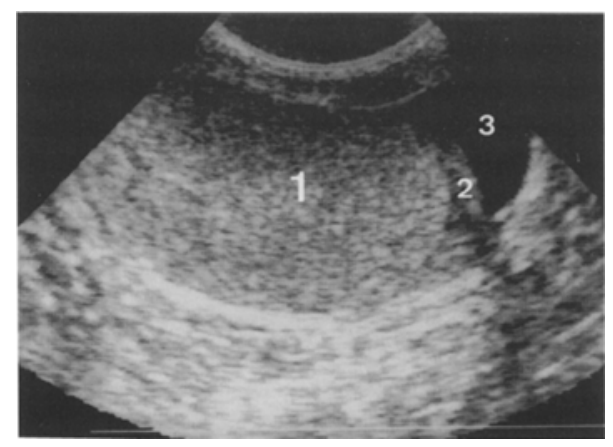

Fig. 1 : Testicule normal - Echographie.

1 - Testicule - 2: Epididyme - 3: Lame liquidienne vaginale 


\section{- la volumétrie prostatique}

Le volume de la prostate peut être calculé. La masse volumique est voisine de l'unité. Le poids peut donc être donné à partir du volume calculé. A l'état normal, la prostate pèse entre 10 et 20 grammes, ce poids a tendance à augmenter en fonction de l'âge. Les mensurations par voie endorectale sont précises, alors que celles obtenues par voie sus-pubienne sont majorées du fait de l'inclinaison du faisceau ultrasonore Les dimensions des vésicules séminales ont peu d'intérêt du fait des grandes variations physiologiques. Elles varient de 3 à $5 \mathrm{~cm}$ de long et de 1 à $2,5 \mathrm{~cm}$ de large (6).

\section{- la structure glandulaire}

Bien que l'on identifie le plus souvent deux zônes dans le parenchyme glandulaire par voie sus-pubienne, et que l'on visualise les vésicules séminales l'analyse est grossière. La voie endorectale permet une analyse beaucoup plus précise des différentes structures. La prostate apparaît comme une glande symétrique de forme ovalaire à grand axe transversal près de la base et arrondie proche de l'apex. Elle est entourée d'un fin liseré hypoéchogène. Chez le sujet jeune on distingue deux parties : l'une est petite, hypoéchogène homogène, de topographie antéro-supérieure au contact de la vessie et correspond à la zone de transition et au stroma fibro-musculaire antérieur.
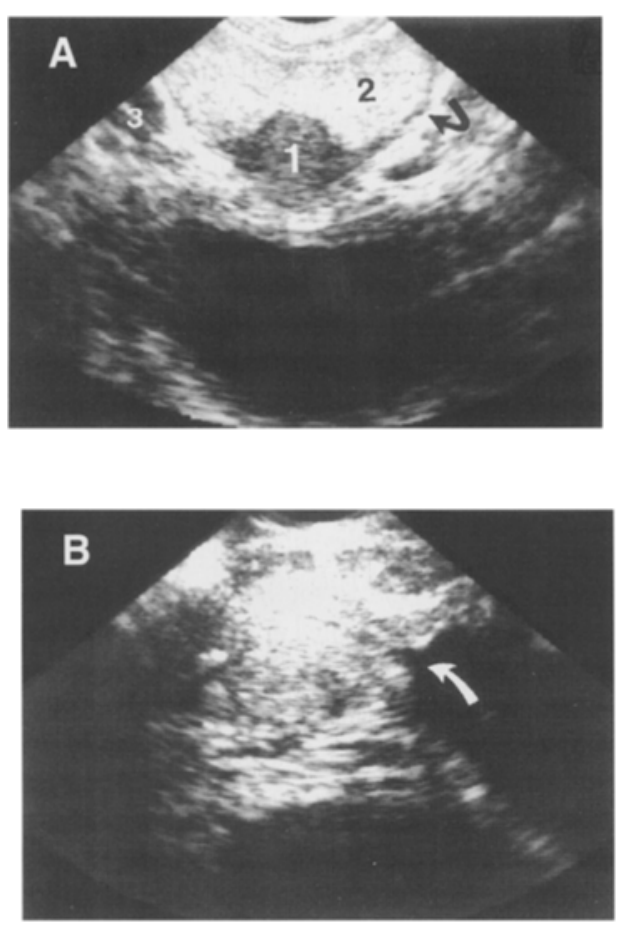

Fig. 2: Prostate nomale du sujet jeune

A : Plan axial - 1 : partie centrale hypoéchogène - 2 : partie périphérique échogène - 3 : plexus vcincux pćriprostatiques. Bande hypoéchogène périphérique $\Omega$

B : Plan sagittal : col vésical

bec de la vésicule séminale. $A$
L'autre est plus volumineuse, échogène, homogène, de topographie postero-inferieure, et correspond à la zone centrale et périphérique (fig. 1). L'urètre et les canaux éjaculateurs sont parfois identifiables sur les coupes supérieures axiales sous forme de 3 zones ponctiformes d'échogénicité différente. La zone de pénétration dans la base de la prostate des canaux éjaculateurs est le plus souvent identifiée dans le plan sagittal par une structure triangulaire hyperéchogène à sommet interne appelé "bec des canaux éjaculateurs"(Fig. 2). En introduisant davantage la sonde, les vésicules séminales apparaissent sous la forme de deux masses hypo ou faiblement échogènes, oblongues ou ovalaires, grossièrement symétriques dans le plan axial et à contours mammelonnés. Parfois elle sont très hypoéchogènes et on identifie des cloisons internes plus échogènes. Les ampoules déférentielles sont deux structures hypo ou faiblement échogènes arrondies, paramédianes mesurant quelques millimètres (inférieures ou égales à 5 millimètres de diamètre).

- étude de l'appareil urinaire par voie sus-pubienne (vessie, reins)

\section{L'uréthrocystographie rétrograde et l'urographie intra-veineuse :}

Elles complètent le bilan d'une pathologie infectieuse ou congénitale car il s'agit souvent d'une atteinte du complexe uro-génital.

\section{La déférentovésiculographie}

C'est la seule méthode d'imagerie qui visualise de façon satisfaisante les canaux déférents, les canaux éjaculateurs et la cavité des vésicules séminales (1). Il s'agit d'un examen invasif, effectué au bloc opératoire sous anesthésie générale. Plusieurs voies sont possibles. Le cathétérisme des canaux éjaculateurs par urétroscopie est une technique difficile. La voie funiculaire par injection de produit de contraste iodé hydrosoluble dans le canal déférent après dissection du cordon à la racine des bourses est plus usuelle. L'injection dans le sens antérograde réalise une déférentovésiculographie. L'injection dans le sens rétrograde, à très faible pression réalise une déférentoépididymographie. Chez l'homme fertile elle est contre indiquée car l'injection à contre courant peut provoquer des fissures de l'épididyme. Des complications infectieuses avec oblitération déférentielle secondaire ont fait la mauvaise réputation de cet examen.

\section{La tomodensitométrie (TDM)}

La tomodensitométrie est peu intéressante pour l'exploration du complexe séminoprostatique car elle est limitée par sa médiocre résolution en contraste, et l'analyse dans un seul plan de l'espace.
Cet examen est inutile pour l'analyse du scrotum. Par contre, la tomodensitométrie est plus performante que l'échographie pour l'étude du canal inguinal, de la cavité pelvienne et du rétropéritoine.

\section{L'IMAGERIE PAR RESONANCE MAGNE- TIQUE (IRM)}

Malgré la résolution inférieure à celle de la TDM, l'IRM est particulièrement intéressante pour l'étude de la prostate et des vésicules séminales. Elle permet une analyse dans les 3 plans de l'espace et possède une excellente résolution en contraste avec individualisation des différentes zones anatomiques. Elle ne nécessite aucune préparation. Il est impératif de respecter les contreindications formelles : présence d'un pacemaker et de matériel métallique intra cérébral. C'est un examen non invasif et qui fournit une iconographie de qualité d'analyse facile pour le correspondant clinicien (5).

\section{IMAGERIE MEDICALE ET PATHOLOGIE DE LA VOIE SEMINALE : \\ Prostatite et vésiculite aigues, abcès}

Le diagnostic des infections aigues de la prostate est avant tout clinique.

L'imagerie médicale a 2 objectifs : au début conforter le diagnostic et dans un second temps suivre l'évolution sous traitement à la recherche d'une complication (abcédation). L'examen échographique occupe la première place. Il sera réalisé par voie sus-pubienne ou périnéale au stade aigu, compte tenu des phénomènes douloureux et du risque de bactériémie.

Le diagnostic échographique de prostatite aiguë repose sur plusieurs éléments associés ou non : augmentation modérée de la taille avec symétrie conservée, contours nets ou mal définis, halo hypoéchogène périurétral, hétérogèneité du parenchyme glandulaire avec zones moins échogènes mal délimitées et élargissement des plexus veineux périprostatiques.

Le diagnostic échographique de vésiculite aigüe repose sur une augmentation nette de la taille de la vésicule avec franche hypoéchogénicité et identification d'une paroi épaisse. Si l'atteinte est bilatérale, il s'agit d'un aspect "boudiné, en massue". La pathologie inflammatoire des ampoules déférentielles se traduit par un élargissement avec hypoéchogénicité. Le plus souvent la prostatite aigue s'accompagne d'une atteinte vésiculaire (3).

A l'inflammation aigue non ou mal traitée peut parfois succéder un stade de collection avec formation d'un abcès. L'abcès prostatique se traduit par une formation arrondie ou ovalaire hypo ou anéchogène avec parfois des cloisons ou des 
secteurs plus échogènes. L'abcès dans la vésicule séminale est identique. Le diagnostic différentiel avec un kyste surinfecté ou hémorragique est impossible sauf si le kyste était antérieurement connu. Les abcès des vésicules séminales sont rares. Ce sont plus souvent des lésions d'échostructure mixte à parois épaisses (6). Le diagnostic d'abcédation est important car il impose une sanction chirurgicale dont la technique diffère selon la topographie de l'abcés ou une ponction évacuatrice échoguidée si l'abcès, n'est pas cloisonné et le pus suffisamment fluide. L'échographie, éventuellement endorectale si cela est possible, est en règle suffisante. La TDM et l'IRM doivent être réalisées si l'on soupçonne une extension extraprostatique périnéale ou pelvienne. Ultérieurement l'échographie contrôle l'évolution qui se fait rarement vers la restitution ad integrum, mais plus souvent vers la calcification.

\section{Lithiase prostatique et vésiculaire}

Les calculs prostatiques forment une entité pathologique fréquente et banale dont la caractéristique est d'être peu ou pas symptomatique. Leur pathogénie est discutée. Il existe deux origines. Certains semblent provenir de la calcification progressive des concrétions "corps amylacés" situés dans les canaux prostatiques ; d'autres se formeraient dans les canaux ou cavités intra parenchymateuses crées par l'infection. Il est impossible de faire la distinction entre ces 2 hypothèses en dehors des cas de volumineuses calcifications occupant manifestement une cavité néoformée avec un antécédent pathologique infectieux. L'aspect échographique est variable :

- zone plus échogène diffuse sans cône d'ombre correspondant à des concrétions partiellement calcifiées

- zone localisée hyperéchogène de dimension variable avec cône d'ombre typique de calcifications.

Elles sont très fréquemment associées à 2 pathologies : l'hyperplasie prostatique bénigne, où ils sont typiquement en périphérie de celle-ci ,et la prostatite chronique. Lorsqu'elles sont petites et peu nombreuses, elles n'ont aucune signification pathologique.

Les calcifications des vésicules séminales sont de 2 types : les calcifications pariétales sont souvent étendues, bilatérales. Elles sont secondaires à des infections diverses : séquelles d'inflammation, diabète, athérome, tuberculose, bilharziose. Les lithiases vésiculaires sont en règle associées à une malformation congénitale ou une infection chronique $(2,8)$.

\section{Prostatite chronique}

La prostate est de taille normale ou légère- ment augmentée, symétrique ou non, mais avec des contours réguliers. La structure glandulaire est globalement plus échogène que la prostate normale, et hétérogène, remaniée par plusieurs types d'anomalie qui prédominent dans la zone périphérique. Des zones plus échogènes correspondraient à de la sclérose. La lithiase de la prostatite chronique est en règle volumineuse et le plus souvent localisée dans les régions postérolatérales de la zone périphérique. C'est un élément important en faveur du diagnostic. Il existe également des nodules hypoéchogènes. Ces derniers rendent le diagnostic différentiel avec le cancer impossible (3)

L'infection chronique des vésicules séminales peut se traduire par une lithiase intra-vésiculaire. Ailleurs, la sténose inflammatoire des canaux éjaculateurs est responsable d'une dilatation kystique des vésicules. Au stade de séquelles la déférentovésiculographie permet de situer l'obstacle.

\section{Pathologie kystique et anomalie congénitale $(7,8,10,11)$}

Le diagnostic de kyste est aisé en échographie et repose sur des critères stricts : masse arrondie ou ovalaire, anéchogène, homogène avec renforcement postérieur et paroi échogène fine et régulière. Avec les sondes endorectales actuelles, la résolution est de 2 à $3 \mathrm{~mm}$. En TDM, il s'agit d'une masse arrondie ou ovalaire de densité liquidienne, non modifiée par l'injection de produit de contraste et sans paroi visible. En IRM la masse a un hyposignal en séquence pondérée en $\mathrm{Tl}$ (SpTl) et un hypersignal en séquence pondérée en $\mathrm{T} 2$ (SpT2).

Toutefois, si le kyste est compliqué d'hémorragie ou d'infection, des échos internes apparaissent, en TDM la densité s'élève ainsi que le signal en IRM sur la SpTl.

Le diagnostic de kyste de l'utricule repose sur la topographie médiane, postérieure, accolée au veru montanum (Fig. 3).

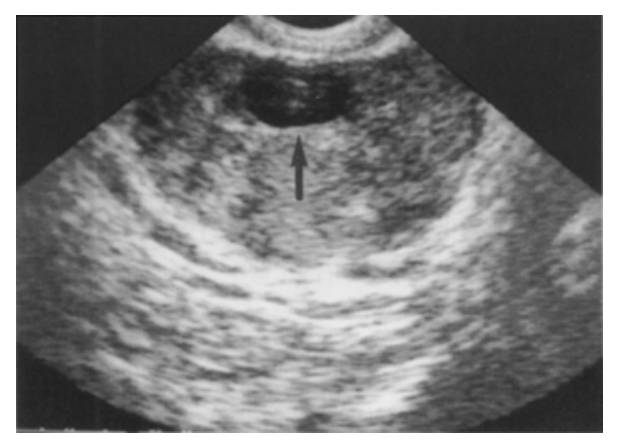

Fig. 3 : Kyste de l'utricule prostatique ( $\uparrow$ ). Echographie endorectale. Remaniements diffus hétérogènes de l'échostructure glandulaire dûs à une prostatite chronique.
S'il est volumineux, il peut comprimer les canaux éjaculateurs, ce qui entraîne des vésicules séminales de grande taille ou parfois franchement hypertrophiée ou encore l'urètre, pouvant aboutir à une vessie de lutte.

Les kystes des canaux éjaculateurs sont latéralisés, primitifs ou secondaires à une obstruction.

Les malformations congénitales des vésicules séminales sont d'un grand polymorphisme. Elles peuvent être isolées ou intéresser plusieurs segments des voies séminales. Elles sont fréquemment associées à des malformations urinaires. Le bilan de ces malformations complexes nécessite le recours à plusieurs méthodes d'imagerie. Examen de première intention, l'échographie sus-pubienne et endorectale affirme l'absence ou l'hypoplasie d'une vésicule, la nature liquidienne d'une masse, et analyse ses parois de facon fiable. Son siège et sa situation par rapport aux organes de voisinage sont correctement précisés si sa taille est modérée. Cependant, lorsque la lésion est volumineuse il peut être impossible d'éliminer un diverticule vésical sans collé visible, un kyste prostatique, une urétérocèle, une duplication digestive ou une néoformation pelvienne intra ou extra-péritonéale.

La radiologie conventionnelle a le rôle primordial pour la poursuite de la démarche diagnostique. L'urographie intraveineuse précise l'anatomie des voies urinaires. L'urétrocystographie rétrograde visualise une communication avec l'urètre mais sa topographie précise par rapport au veru montanum est parfois incertaine. Une ponction échoguidée du kyste a l'avantage de mettre en évidence du liquide spermatique (par opposition aux kystes utriculaire et prostatique) et après opacification une communication avec les voies séminales et/ou urinaires. La déférentovésiculographie permet un bilan complet des voies séminales avec classification de l'anomalie. Elle sera complétée par une urétroscopie.

L'IRM a un intérêt morphologique incontestable grâce au plan sagittal et frontal surtout pour les grosses masses pour lesquelles l'échographie endorectale est insuffisante (Fig. 4).

Les kystes acquis représentent la pathologie tumorale la plus fréquente au niveau des vésicules séminales. Ils sont médians ou latéralisés, le reste de la vésicule étant normal. Ils contiennent fréquemment des sédiments intrakystiques et peuvent se compliquer d'hémorragie ou d'infection. Quelque soit le type de complication, cela se traduit par des échos déclives ou diffus intrakystiques et en IRM un signal modéré à élevé mais inférieur à celui de la graisse en $\mathrm{SpTl}$. 

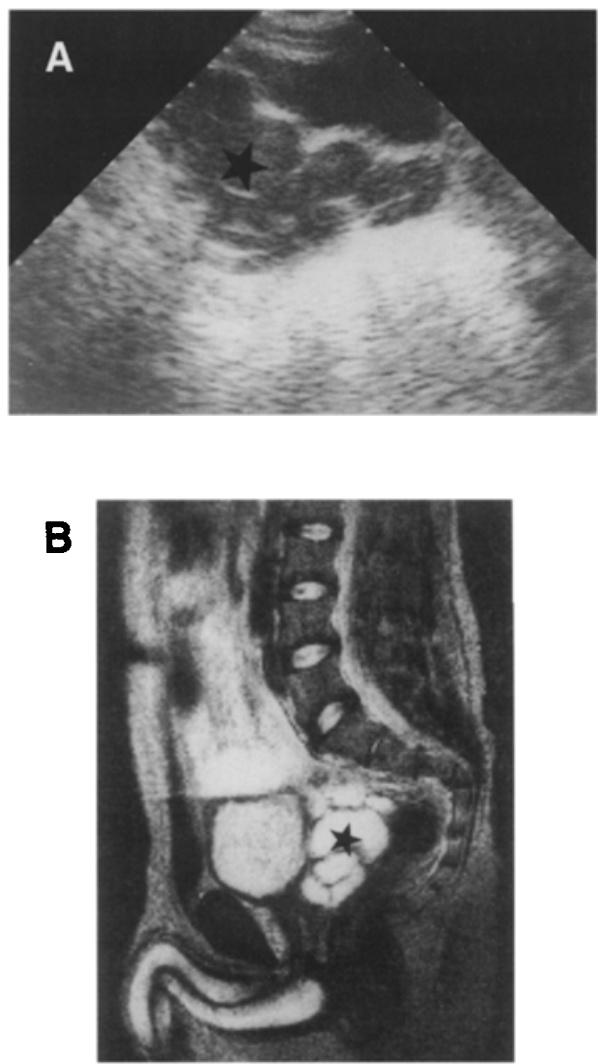

Fig. 4 : Dystrophie kystique des vésicules séminales

A : Echographie sus-pubienne - dilatation kystique des vésicules $\left(^{*}\right.$ ) B : IRM - SpT2 - plan sagittal - hypersignal du contenu (*) dû à sa nature liquidienne.

\section{Hemospermie}

Ce symptome rare, et dans la majorité des cas bénin, est d'étiopathogénie inconnue. Bien qu'il existe de multiples étiologies, les formes idiopathiques sont fréquentes. En pratique, la découverte d'une étiologie est proportionnelle aux perfectionnements des méthodes d'explorations (9). L'échographie endorectale a montré une association significative entre hémospermie et calculs dans les vésicules séminales, les canaux éjaculateurs et les canaux prostatiques autour de l'urètre périmontanal. L'échographie endorectale permet également d'identifier des varicosités périprostatiques et périvésiculaires. L'IRM est la seule méthode d'imagerie qui objective le saignement.

\section{Orchiépididymite aigue}

C'est la forme la plus fréquente d'inflammation aigue des bourses. L'aspect échographique associe de façon plus ou moins complète un épaississement des enveloppes scrotales, hypertrophie hétérogène localisée ou diffuse de l'épididyme et du testicule. Devant un tableau clinique sévère, il faudra rechercher des signes échographiques de gravité : intensité de l'hypoéchogénicité testiculaire, surtout au niveau du hile, et importante hypertrophie de l'épididyme. Ces éléments font craindre une ischémie aigue du testi- cule dûe à la compression du hile vasculaire par l'épididyme. Elle devra être levée chirurgicalement. L'évolution peut se faire vers l'abcédation. Il s'agit d'une masse arrondie ou ovalaire intra ou extratesticulaire, anéchogène ou hypoéchogène mal limitée (contenant des échos grossiers et des cloisons), dans un testicule hypertrophié.

Après traitement, la guérison complète se traduit par une restitution ad integrum. Ailleurs, il persistera des séquelles: testicules de petite taille avec échogénicité plus faible, hétérogène avec parfois des calcifications.

En cas d'épididymite chronique localisée, il existe un noyau épididymaire échogène à hyperéchogène, avec parfois des calcifications au niveau de l'anse épididymo-déférentielle ou plus rarement une zone liquidienne multicloisonnée. L'atteinte diffuse est possible avec hétérogénéité ou aspect polylobé hyperéchogène.

\section{Les dystrophies kystiques de l'épididyme}

Elles peuvent être d'origine congénitales (kyste de l'épididyme) ou acquises (spermatocèle) en réaction à une inflammation de l'épididy. me.

Le diagnostic échographique est facile devant une formation arrondie ou ovalaire, unie ou polylobée de taille variable, anéchogène avec renforcement postérieur à paroi fine et régulière. Sa topographic est intra ou paraépididymaire au niveau de la tête le plus souvent. La distinction entre kyste de l'épididyme et spermatocèle est impossible.

Les kystes du cordon responsable d'une grosse bourse sont plus rares. Il s'agit d'une formation anéchogène située au-dessus de l'épididyme et du testicule.

\section{Torsion aigue du cordon}

Le diagnostic de torsion aigue du cordon est clinique et l'intervention exploratrice s'impose au moindre doute.

En phase tardive, séquellaire, le testicule s'atrophie et sa structure devient hétérogène avec des plages hypo et hyperéchogènes.

L'échographie Doppler couleur permet le diagnostic différentiel entre torsion et orchiépididymite. En cas de torsion, la vascularisation testiculaire disparaît complètement. Mais l'imagerie médicale ne doit en aucun cas retarder le geste chirurgical.

\section{Varicocèle}

La dilatation variqueuse des veines du plexus pampiniforme antérieur est de diagnostic échographique facile (Fig. 5) et repose sur trois types d'arguments :

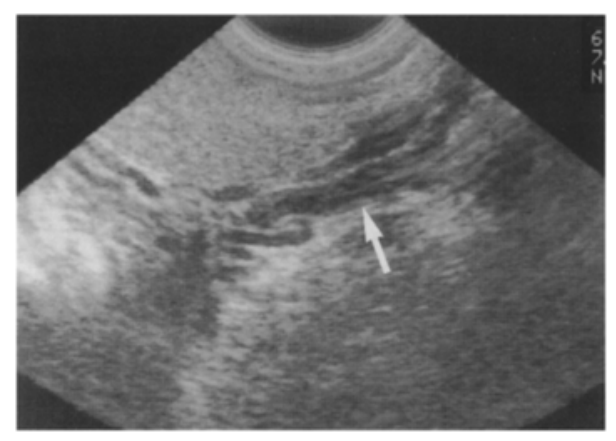

Fig. 5 : Varicocèle - Echographie :

Dilatation serpigineuse des veines $(\uparrow)$.

- réseau anéchogène tubulaire serpigineux et tortueux, chaque élément ayant quelques millimètres de diamètre.

- de siège postéro-supérieur en arrière de l'épididyme et du testicule, se prolongeant en haut dans le cordon

- augmentation du calibre lors des manoeuvres dynamiques (manoeuvre de Valsalva et orthostatisme). Il est facilement comprimé par la sonde.

Il est plus fréquent à gauche. Le diagnostic est facilité par l'examen Doppler couleur qui permet de mettre en évidence des structures veineuses circulantes, même de petite taille, soit spontanément, soit au cours des manoeuvres dynamiques. Il faut rechercher un retentissement sur le testicule, qui se traduit par une diminution de taille avec une échostructure normale ou hypoéchogène. Ceci témoigne de la sévérité de la varicocèle.

Il faut compléter l'examen par la recherche d'une cause rétropéritonéale responsable d'une thrombose veineuse, bien que ce soit une étiologie peu fréquente.

\section{Ectopie testiculaire et cryptorchidies}

Le diagnostic de bourse vide est facile à l'examen clinique ou en échographie. Le diagnostic topographique fait intervenir plusieurs méthodes d'imagerie. L'échographie est plus performante pour l'exploration inguinale superficielle que profonde, d'autant plus que le testicule en situation anormale est toujours atrophique (variant de 1 à $2 \mathrm{~cm}$ ) et hypoéchogène. Pour les autres localisations c'est la tomodensitométrie et/ou l'imagerie par résonnance magnétique qui sont utiles. La tomodensitométrie est performante pour le canal inguinal, mais peut être d'analyse difficile autour des axes vasculaires pelviens. L'imagerie par résonance magnétique offre l'avantage du plan de coupe frontal ou sagittal avec évaluation de la distance qui sépare le testicule des différents orifices du canal inguinal. Mais elle ne permet pas dans le même examen de réaliser une exploration abdominale haute. De toute façon, quelle que soit la méthode utilisée, le 
risque de faux négatif est dû à l'atrophie testiculaire. Il faudra compléter le bilan par la recherche d'une malformation congénitale associée urogénitale.

\section{CONCLUSION}

L'exploration des voies séminales s'est enrichie de techniques non invasives qui permettent l'étude des parenchymes. Les structures canalaires, surtout lorsqu'un geste chirurgical est envisagé, demeurent du domaine de la radiologie conventionnelle.

L'échographie transrectale a connu une grande diffusion depuis quelques années, surtout dans le dépistage du cancer de la prostate, malgré toutes les difficultés rencontrées. C'est également le premier examen à effectuer pour l'étude des glandes sexuelles accessoires. Sa résolution permet une approche anatomique très rigoureuse. L'IRM est une méthode très intéressante pour la morphologie et la caractérisation du contenu des liquides. Mais elle demeure un examen coûteux, long et n'est disponible que dans certains centres. La déférentovésiculographie reste un examen invasif mais c'est encore la référence pour le diagnostic des obstructions de la voie séminale.

\section{REFERENCES}

1- Boreau J. : Les images des voies séminales / Images of the seminal tracts. S. Karger Ed., Bale, 1974
2- Carter S.S., Shinohara K, Lipshultz L.I. : Transrectal ultrasonography in disorders of the seminal vesicles and ejaculatory ducts. Urol. Clin. North. Amer, 1989 , 16: 773 - 790

3 - Doble A, Carter S.S : Ultrasonography findings in Prostatitis. Urol. Clin. North Amer, 1989, 16 : $763-772$

4- Fornage B : Echographie de la prostate. Vigot, Paris, 1985

5 - Hricak H, Carrington B.M. : MRI of the Pelvis The Seminal Vesicles, Dunitz, 1991 : 313 - 318

6- King B.F, Hattery R.R, Lieber M.M, Williamson B, Hartmann G.W, Thomas H.B : Seminal Vesicle Imaging Radiographics, 1989 , 9:653 - 675

7 - Kneelano J.B, Auh Y.H, Mc Carron J.P, Zirinsky K, Rubeinstein W.A, Kazam E : Computed Tomography, Sonography, Vesiculography and MRI Imaging of a Semina! Vesicle Cyst. J. Comput. Assist. Tomogr, 1985 , 9:964-966

8 - Littrup P.J, Lee F, Mc Cleary R.J, Wu D, Lee A, Kumasata $G$ Transrectal US of the seminal vesicle and ejaculatory ducts : clinical correlation. Radiology, 1988, 168: 625-629.

9- Marshall V.F, Fuller N.L : Hemospermia. J. Urol, 1983, 129: $377-378$.

10 - Shabsigh R, Lerner S, Fishman I : The role of transrectal ultrasound in the diagnosis and management of prostatic and seminal vesicle cysts. J. Urol, 1989 , 14: 1206 - 1209
11 - Von Poppel H, Verduyn H, Verrecken R, De Geeter $\mathrm{P}$ : Kystes congénitaux de la prostate Acta Urol Belg, 1982 , $50: 359$ - 364.

RESUME : Les méthodes d'imagerie médicale pour l'exploration des voies séminales se sont multipliées ces dernières années. L'examen échographique, surtout grâce au développement des sondes de haute fréquence et endocavitaires, permet une approche morphologique trés précise des parenchymes, mais il n'explore pas les structures canalaires dont l'analyse est encore du domaine de la radiologie conventionnelie. Les méthodes d'imagerie dites "lourdes", tomodensitométrie et imagerie par résonance magnétique, ont des indications plus limitées. Dans tous les cas, les résultats de l'imagerie doivent être confrontés à ceux de l'examen clinique et des résultats biologiques. Mots clés : Echographie testiculaire, Echographie endorectale, IRM, Vésicules Séminales, Prostate. Andrologie, 1992, 2 : 70-74. 